\title{
Differentiation between multiple sclerosis and neuromyelitis optica spectrum disorder
}

\author{
Authors: Anamaria Dukić ${ }^{1}$, Nikola Erceg ${ }^{1}$, Tereza Gabelić ${ }^{1,2}$ (mentor) \\ ${ }^{1}$ School of Medicine, University of Zagreb, Croatia \\ ${ }^{2}$ University Hospital Centre Zagreb, Departement of Neurology, Croatia \\ DOI: https://doi.org/10.26800/LV-142-supp5-17
}

\section{Background:}

Multiple sclerosis (MS) can sometimes be misdiagnosed with neuromyelitis optica spectrum disorder (NMOSD). NMOSD is a chronic disorder of the brain and spinal cord dominated by inflammation of the optic nerve (optic neuritis) and inflammation of the spinal cord (myelitis).

\section{Case presentation:}

A 54-year-old female patient first presented in 2007 with weakness of the right leg that lasted for one month. In 2012 she had weakness of both legs and arms. In 2015 she had bilaterally positive Babinski and was diagnosed clinically with spastic tetraparesis. She had a spastic gait and urge incontinence as well. Magnetic resonance imaging (MRI) of the brain and cervical medulla was atypical for MS. It demonstrated multiple hypertensive lesions in the brainstem; in the area of the bottom of IV ventricles, pons, left upper cerebellar peduncle, and in the mesencephalon. There were lesions in the left cerebellar hemisphere, in the area of the basal ganglia, and periventricular regions. Longitudinal extensive confluent lesions were also found from $\mathrm{C} 1$ to $\mathrm{C} 7$ vertebral segments of the cervical medulla. Lumbar puncture revealed oligoclonal bands type two (intrathecal synthesis), and positive MRZ (measles-rubella-varicella-zoster virus) reaction. The anti-aquaporin-4 antibodies (NMO antibodies) were positive in 3 consecutive testings (ELISA method), which are often associated with NMOSD. Samples were sent to Mayo Clinic for cell based assay testing in 2016, and results were negative. It was concluded that the patient has primary progressive MS with false-positive results of ELISA testing. Treatment with ocrelizumab was introduced. The patient is clinically stable, without treatment side effects, and with no disease activity on MRI.

\section{Conclusion:}

It is crucial to have a broad spectrum of clinical, laboratory, and MRI examinations because misdiagnosis of the demyelinating disease can lead to wrong therapy and worsening of the disease.

Keywords: Lesions, Multiple sclerosis, Neuromyelitis optica spectrum disorder 